\title{
Influence of boundary conditions on statistical properties of ideal Bose-Einstein condensates
}

\author{
Martin Holthaus* \\ Fachbereich Physik, Carl von Ossietzky Universität, D-26111 Oldenburg, Germany \\ Kishore T. Kapale and Marlan O. Scully \\ Max-Planck-Institut für Quantenoptik, D-85748 Garching, Germany \\ and Department of Physics and Institute for Quantum Studies, Texas A\&M University, College Station, Texas 77843
}

(Received 23 October 2001; published 27 February 2002)

\begin{abstract}
We investigate the probability distribution that governs the number of ground-state particles in a partially condensed ideal Bose gas confined to a cubic volume within the canonical ensemble. Imposing either periodic or Dirichlet boundary conditions, we derive asymptotic expressions for all its cumulants. Whereas the condensation temperature becomes independent of the boundary conditions in the large-system limit, as implied by Weyl's theorem, the fluctuation of the number of condensate particles and all higher cumulants remain sensitive to the boundary conditions even in that limit. The implications of these findings for weakly interacting Bose gases are briefly discussed.
\end{abstract}

DOI: 10.1103/PhysRevE.65.036129

PACS number(s): 05.30.Ch, 05.30.Jp, 03.75.Fi

When London, in 1938, wrote his now-famous papers on Bose-Einstein condensation of an ideal gas [1,2], he simply considered a free system of $N$ noninteracting Bose particles without an external trapping potential, imposing periodic boundary conditions on a cubic volume $V$. The rationale for so doing lies in Weyl's theorem [3]. In the thermodynamic limit the particular boundary conditions are not supposed to play any role. Yet, for any box size the half-sine singleparticle ground state in a box with hard walls is quite different from the plane-wave ground state in a box with periodic boundary conditions, so that, intuitively, one expects some influence of the boundary conditions on the physics of BoseEinstein condensation even in the large-system limit (that is, for $N \rightarrow \infty$ and $V \rightarrow \infty$, such that the density $N / V$ remains constant). In this paper we will explain that, indeed, all "higher" statistical properties of an ideal, free Bose-Einstein condensate, such as the fluctuation $[4,5]$ of the number of condensed particles, remain sensitive to the boundary conditions even in the large-system limit, even though the condensation temperature does not. We speculate, however, that this unusual behavior constitutes a pathology of the ideal gas that will be cured by interparticle interactions, although so far no detailed proof for this surmise exists.

Let us consider an ideal, $N$-particle Bose gas stored in some trap with single-particle energies $\varepsilon_{\nu}(\nu=0,1,2, \ldots$; in general, $\nu$ abbreviates a multi-index). We assume further that the setup conforms to the canonical ensemble, which means that the trapped gas is kept in thermal contact with an external heat bath of temperature $T$. The starting point of our analysis is the recognition that for temperatures below the onset of Bose-Einstein condensation the canonical partition function $\mathcal{Z}(\beta)$ reduces, to excellent approximation [6], to the partition function of a system of infinitely many independent (i.e., Boltzmannian) harmonic oscillators,

$$
\mathcal{Z}(\beta)=\prod_{\nu=1}^{\infty} \frac{1}{1-\exp \left[-\beta\left(\varepsilon_{\nu}-\varepsilon_{0}\right)\right]},
$$

*Electronic address: holthaus@marvin.physik.uni-oldenburg.de with $\beta=1 /\left(k_{\mathrm{B}} T\right)$, where $k_{\mathrm{B}}$ denotes Boltzmann's constant. Note that the product runs over the excited states only, excluding the ground state $\nu=0$; the frequencies of the individual oscillators being given by the excited-states energies $\varepsilon_{\nu}$ relative to the ground-state level $\varepsilon_{0}$. Note further that the equivalence of a partially condensed ideal Bose gas and a harmonic-oscillator system holds regardless of the precise form of the single-particle spectrum, so that it does not matter whether or not the trapping potential itself is harmonic.

The only approximation entering into the derivation of the canonical partition function (1) is a replacement of the actual, finite number of condensed particles by a condensate containing infinitely many particles [6]. For temperatures such that a sizeable fraction of the particles is condensed, this enlargement of the condensate does not appreciably change the thermal properties of the system. On the other hand, assuming an inifinite supply of condensed particles means excluding the onset of condensation from the analysis; this onset can be treated by a different approach based on a master equation [4,5]. In short, the validity of Eq. (1) is restricted to the condensate regime. The same approximation had already been used by Fierz in 1956 [7] for computing the fluctuation of an ideal Bose-Einstein condensate; it also underlies the "Maxwell's demon ensemble" suggested by Navez et al. [8]. In a different guise, it has been utilized in the canonical quasiparticle approach formulated by Kocharovsky, Kocharovsky, and Scully $[9,10]$.

Having stepped from the original trapped Bose gas to a harmonic-oscillator system, we now exploit the fact that there exist powerful mathematical tools for evaluating harmonic-oscillator sums [11] in order to quantify the statistics of the ground-state occupation number of the partially condensed Bose gas described by Eq. (1). Specifically, we study the canonical probability distribution $p_{N}\left(N_{\mathrm{ex}} ; \beta\right)$ for finding $N_{\text {ex }}$ of the $N$ particles in an excited state at a given inverse temperature $\beta$, so that the number of condensate particles is $n_{0}=N-N_{\mathrm{ex}}$. This distribution is characterized by its cumulants $\kappa_{k}(\beta)[9,10]$. The first cumulant $\kappa_{1}(\beta)$ is the canonical-ensemble expectation value $\left\langle N_{\text {ex }}\right\rangle$ of the number 
of excited particles, the second cumulant $\kappa_{2}(\beta)$ corresponds to the mean-square fluctuation of $N_{\mathrm{ex}}$ (thus equalling the fluctuation of the number of ground-state particles), $\kappa_{3}(\beta)$ equals the third central moment $\mu_{3}(\beta)$; similarly, $\kappa_{4}(\beta)$ $=\mu_{4}(\beta)-3 \kappa_{2}(\beta)^{2}[12]$. The reason for focusing on the cumulants $\kappa_{k}(\beta)$, rather than on the more familiar central moments $\mu_{k}(\beta)$, lies in the independence of the Boltzmannian oscillators: The $k$ th order cumulant of a sum of independent stochastic variables is given by the sum of the individual cumulants.

Without any further approximation, one can then derive a compact integral representation for $\kappa_{k}(\beta)$. Introducing the generalized zeta function

$$
Z(\beta, t) \equiv \sum_{\nu=1}^{\infty} \frac{1}{\left(\beta\left[\varepsilon_{\nu}-\varepsilon_{0}\right]\right)^{t}},
$$

we find [6]

$$
\kappa_{k}(\beta)=\frac{1}{2 \pi i} \int_{t_{0}-i \infty}^{t_{0}+i \infty} \mathrm{d} t \Gamma(t) Z(\beta, t) \zeta(t+1-k) .
$$

Here, $\Gamma(t)$ and $\zeta(t)$ denote the gamma function and the Riemann zeta function, respectively; the real number $t_{0}$ has to be chosen such that the path of integration parallel to the imaginary axis of the complex $t$ plane lies to the right of all poles of the integrand. Note that the properties of the particular trap (that is, the single-particle spectrum) enter into $\kappa_{k}(\beta)$ only through $Z(\beta, t)$, whereas the cumulant order $k$ is determined only by the argument of the Riemann zeta function. The usefulness of this cumulant formula (3) stems from the fact that there exist well-established techniques [13] for continuing the "spectral" zeta functions (2) analytically to the complex $t$ plane, so that simply collecting the residues at the poles of the product $\Gamma(t) Z(\beta, t) \zeta(t+1-k)$, taken from right to left, results in a systematic asymptotic expansion of the desired canonical cumulants $\kappa_{k}(\beta)$. The large-system limit is governed by the rightmost pole alone [6].

We now apply these general findings to an ideal Bose gas of $N$ particles with mass $m$ in a cubic volume $V=L^{3}$. Imposing periodic boundary conditions on the wave functions, as London did [1,2], and defining the frequency

$$
\Omega \equiv \frac{\hbar(2 \pi)^{2}}{2 m L^{2}},
$$

the spectral zeta function (2) then acquires the form

$$
Z(\beta, t)=(\beta \hbar \Omega)^{-t} S(t) .
$$

Here,

$$
S(t) \equiv \sum_{n_{1}, n_{2}, n_{3}=-\infty}^{+\infty} \frac{1}{\left(n_{1}^{2}+n_{2}^{2}+n_{3}^{2}\right)^{t}}
$$

is a zeta function of the Epstein type $[14,15]$. The sum runs over all eight octants of quantum numbers $\left(n_{1}, n_{2}, n_{3}\right)$; the prime indicating that the ground state with energy $\varepsilon_{0}=0$ has to be excluded, in obeyance of the general prescription (2).
Analytically continuing this function $S(t)[13,14]$, one finds that it possesses merely one simple pole, located at

$$
t=3 / 2 \text { with residue } 2 \pi \text {. }
$$

For $k=1$ this pole of $Z(\beta, t)$ lies to the right of the pole of $\zeta(t)$ at $t=1$ [16], and, thus, dominates the temperature dependence of the number of excited particles. Defining the scaled temperature $\tau \equiv k_{\mathrm{B}} T /(\hbar \Omega)$, the canonical-ensemble expectation value $\left\langle N_{\mathrm{ex}}\right\rangle=\kappa_{1}(\beta)$ of the number of excited particles then takes, for $\tau \gg 1$, the form

$$
\left\langle N_{\mathrm{ex}}\right\rangle \sim \pi^{3 / 2} \zeta(3 / 2) \tau^{3 / 2}+S(1) \tau,
$$

with $S(1) \approx-8.914$. This asymptotic expression, valid as long as $\left\langle N_{\mathrm{ex}}\right\rangle \leqslant N$ (that is, in the condensate regime), yields excellent agreement with exact numerical data even for merely moderately large particle numbers [6].

In contrast, for all higher cumulants, $k \geqslant 2$, it is no longer the pole (7) of $Z(\beta, t)$ that dominates $\kappa_{k}(\beta)$ in the asymptotic regime $\tau \gg 1$, but rather the pole of $\zeta(t+1-k)$ at $t=k$. Hence, to leading order $\kappa_{k}(\beta)$ becomes proportional to $\tau^{k}:$

$$
\kappa_{k}(\beta) \sim(k-1) ! S(k) \tau^{k}+\pi^{3 / 2} \zeta(5 / 2-k) \tau^{3 / 2} .
$$

In the next step we repeat this analysis for the case of Dirichlet boundary conditions, that is, for an ideal $\mathrm{N}$-particle Bose gas stored in a cubic volume $V=L^{3}$ with hard, impenetrable walls. This implies a nonzero ground-state energy $\varepsilon_{0}=3 \hbar \Omega / 4$, so that the corresponding generalized zeta function (2) becomes

$$
Z(\beta, t)=(\beta \hbar \Omega / 4)^{-t} \widetilde{S}(t),
$$

with a modified, inhomogeneous Epstein function $[14,15]$

$$
\widetilde{S}(t) \equiv \sum_{n_{1}, n_{2}, n_{3}=1}^{\infty} \frac{1}{\left(n_{1}^{2}+n_{2}^{2}+n_{3}^{2}-3\right)^{t}} .
$$

In contrast to the previous sum (6), $\widetilde{S}(t)$ comprises only the first octant of triples $\left(n_{1}, n_{2}, n_{3}\right)$, excluding the ground state $(1,1,1)$. This function exhibits simple poles at [6]

$$
t=3 / 2,1,1 / 2 \text { with residues } \frac{\pi}{4},-\frac{3 \pi}{8}, \frac{3+3 \pi}{8} .
$$

Further poles are located at negative half-integer $t$; the higher-order corrections stemming from these additional poles will be neglected here.

When evaluating the cumulant formula (3) for $k=1$, one now encounters a double pole at $t=1$. This forces us to retain also the finite part $\delta$ of $\widetilde{S}(t)$ at $t=1$,

$$
\widetilde{S}(t) \approx-\frac{3 \pi / 8}{t-1}+\delta \quad \text { for } t \approx 1 .
$$

One then finds for the number of excited particles 


$$
\begin{aligned}
\left\langle N_{\text {ex }}\right\rangle \sim & \pi^{3 / 2} \zeta(3 / 2) \tau^{3 / 2}-[(3 \pi / 2) \ln (4 \tau)-4 \delta] \tau \\
& +(3 / 4)(1+\pi) \sqrt{\pi} \zeta(1 / 2) \tau^{1 / 2}
\end{aligned}
$$

the higher $(k \geqslant 2)$ cumulants become

$$
\begin{aligned}
\kappa_{k}(\beta) \sim & 4^{k}(k-1) ! \widetilde{S}(k) \tau^{k}+\pi^{3 / 2} \zeta(5 / 2-k) \tau^{3 / 2} \\
& -\frac{3 \pi}{2} \zeta(2-k) \tau+\frac{3}{4}(1+\pi) \sqrt{\pi} \zeta(3 / 2-k) \tau^{1 / 2}
\end{aligned}
$$

These results allow us to compare the canonical statistics of an ideal condensate in a "periodic box" in detail to that of its Dirichlet counterpart. First we note that $\left\langle N_{\text {ex }}\right\rangle$ tends to be lower in the case of hard walls than in the case of periodic boundary conditions, although both expressions (8) and (14) coincide to leading order in $\tau$. The (logarithmic) reduction of $\left\langle N_{\text {ex }}\right\rangle$ reflects the fact that there are effectively fewer states available in the hard box-when going from the hard to the periodic box by taking eight times the states falling into the first octant of quantum numbers $\left(n_{1}, n_{2}, n_{3}\right)$, the states with one or two of the $n_{i}$ equal to zero are still missing - while the equality of the leading-order terms is in accordance with Weyl's theorem on the spectrum of the Laplacian [3]. That theorem states that the density of states $\rho(E)$ becomes independent of the particular boundary conditions when taking the large-system limit, so that also those quantities that are determined by $\rho(E)$ inherit this independence. In our case, approaching the large-system limit means considering small $\Omega$, and hence large $\tau$. A little reflection reveals that in the large-system limit a cumulant $\kappa_{k}(\beta)$ can be expressed in terms of $\rho(E)$ only if the rightmost pole in Eq. (3) is provided by $Z(\beta, t)$, rather than by the Riemann zeta function. As we have shown, this applies to $k=1$ only. Only the first cumulant $\kappa_{1}(\beta)$ falls into the realm of Weyl's theorem; all higher cumulants remain sensitive to the boundary conditions even in the large-system limit.

The asymptotic (large $\tau$ ) equality of $\kappa_{1}(\beta)=\left\langle N_{\mathrm{ex}}\right\rangle$ for periodic and Dirichlet boundary conditions results in an unambiguous definition of the condensation temperature $T_{0}$, obtained by setting $\left\langle N_{\text {ex }}\right\rangle=N$. Keeping only the respective leading-order term, both Eqs. (8) and (14) give

$$
T_{0}=\frac{\hbar \Omega}{\pi k_{\mathrm{B}}}\left(\frac{N}{\zeta(3 / 2)}\right)^{2 / 3}=\frac{(2 \pi \hbar)^{2}}{2 \pi m k_{\mathrm{B}}}\left(\frac{N}{V \zeta(3 / 2)}\right)^{2 / 3} .
$$

This expression agrees with the familiar textbook result [17] that is usually derived within the grand canonical ensemble, instead of the canonical ensemble employed here.

In contrast, for $k \geqslant 2$ the cumulants $\kappa_{k}(\beta)$, computed with the convenient periodic boundary conditions, differ from their hard-wall counterparts even in the large-system limit. Comparison of Eqs. (9) and (15) shows that the difference is quantified by the temperature-independent ratio $R(k)$ $\equiv 4^{k} \widetilde{S}(k) / S(k)$. Some numerical values of the Epstein sums are listed in Table I; the ratio $R(k)$ is depicted in Fig. 1. Thus, the canonical mean-square fluctuation $\kappa_{2}(\beta)$ of the number of condensate particles in a box with hard walls
TABLE I. Numerical values of the Epstein sums (6) and (11), together with the ratio $R(k)$.

\begin{tabular}{cccc}
\hline \hline$k$ & $S(k)$ & $4^{k} \widetilde{S}(k)$ & $4^{k} \widetilde{S}(k) / S(k)$ \\
\hline 2 & 16.532 & 14.297 & 0.865 \\
3 & 8.402 & 9.312 & 1.108 \\
4 & 6.946 & 10.506 & 1.513 \\
5 & 6.426 & 13.208 & 2.055 \\
6 & 6.202 & 17.193 & 2.772 \\
7 & 6.098 & 22.683 & 3.720 \\
8 & 6.048 & 30.099 & 4.976 \\
9 & 6.024 & 40.040 & 6.647 \\
10 & 6.012 & 53.329 & 8.871 \\
\hline \hline
\end{tabular}

amounts to only $86.5 \%$ of the corresponding fluctuation for the case of periodic boundary conditions; for $k \geqslant 3$, the "hard" cumulant exceeds the "periodic" one.

The excited-particles distribution $p_{N}\left(N_{\mathrm{ex}} ; \beta\right)$ can be characterized further by its skewness [12],

$$
S(\beta)=\kappa_{3}(\beta) / \kappa_{2}(\beta)^{3 / 2}
$$

and the flatness [12]

$$
F(\beta)=3+\kappa_{4}(\beta) / \kappa_{2}(\beta)^{2} .
$$

Figure 2 shows exact, numerically computed [18] values of $-S(\beta)$ for a gas consisting of $N=1000$ ideal bosons, for both periodic and Dirichlet boundary conditions (full lines), and compares these exact data to the approximations provided by the asymptotic expressions (9) and (15), respectively (dashed lines). [We choose to plot the negative of the skewness (17), as corresponding to the third central moment $\left\langle\left(n_{0}-\left\langle n_{0}\right\rangle\right)^{3}\right\rangle=-\kappa_{3}(\beta)$ of the number $n_{0}$ of condensate particles.] The crossover at $T / T_{0} \approx 1$ from the condensate regime to the high-temperature regime, where there is no condensate, lies outside the scope of these asymptotic results, as a consequence of the fiction of an infinite supply of condensate particles that underlies the cumulant formula (3), but in the condensate regime the agreement is close to perfect. It is interesting to note that in both cases the negative skewness stays well below the Gaussian value $-S(\beta) \equiv 0$,

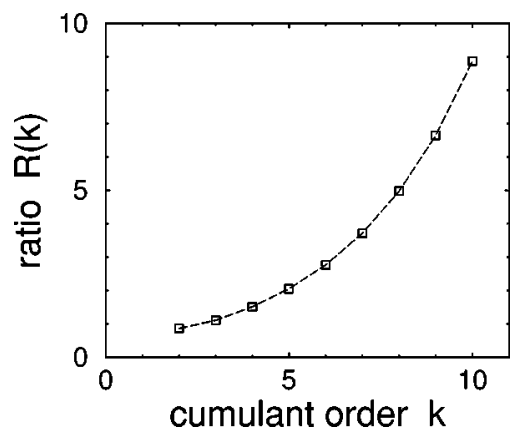

FIG. 1. Ratio $R(k)$ of the canonical cumulant $\kappa_{k}(\beta)$ for an ideal Bose gas enclosed in a cubic container with hard walls to the same cumulant as obtained with periodic boundary conditions. These data refer to the large-system limit. 


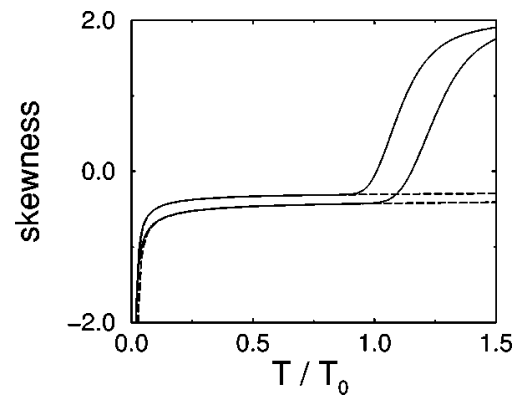

FIG. 2. Exact, numerically computed skewness $-S$ of the distribution $p_{N}\left(N_{\mathrm{ex}} \beta\right)$ for a gas with $N=1000$ ideal bosons in a cubic volume with periodic or Dirichlet boundary conditions (full lines, in the Dirichlet case the crossover to the condensate regime occurs at slightly higher temperatures), compared to the asymptotic approximations (9) and (15) (dashed lines). Note that in both cases the skewness differs sizeably from the Gaussian value $-S=0$. (Periodic, $-S \rightarrow-0.2500$; Dirichlet, $-S \rightarrow-0.3445$.) This is no finitesize effect.

and that the skewness for the Dirichlet box is substantially different from that for the periodic one. This is no finite-size effect. As can be inferred from Table I, in the large-system limit $-S(\beta)$ approaches the constant value -0.2500 in the periodic case, but -0.3445 under Dirichlet boundary conditions.

Figure 3 displays the corresponding data for the flatness. Again, in the condensate regime there is a sizeable difference between the two cases: Periodic bounday conditions lead to $F(\beta) \approx 3.1525$ in the large-system limit, Dirichlet ones to $F(\beta) \approx 3.3084$. Thus, in either case the fluctuations of the number of condensate particles for an ideal Bose gas in a box are essentially non-Gaussian, even in the large-system limit $[9,10]$. This is different from the behavior of an ideal Bose gas in a harmonic-oscillator trap, where skewness and flatness approach the Gaussian values $S=0$ and $F=3$.

The question then arises to which extent this remarkable sensitivity to the boundary conditions exhibited by the ideal Bose gas is shared by a gas with weak interaction among the particles. For an interacting, homogeneous gas with periodic boundary conditions, the condensate fluctuations $[19,20]$ and all higher cumulants $[9,10]$ have already been evaluated with the help of standard Bogoliubov theory. In particular, it has

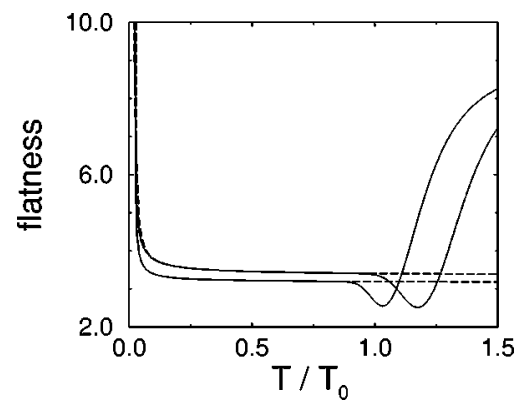

FIG. 3. As Fig. 2, now for the flatness $F$. In both cases, the flatness stays well above the Gaussian value $F=3$ in the condensate regime (periodic, $F \rightarrow 3.1525$; Dirichlet, $F \rightarrow 3.3084$ ). Again, this is no finite-size effect. been shown that in this case the condensate fluctuations are reduced by a factor of 2 as compared with the ideal gas, as a consequence of the pairwise Bogoliubov coupling between single-particle states with wave vectors $\vec{k}$ and $-\vec{k}$. When Dirichlet boundary conditions are imposed, the gas becomes inhomogeneous, and the particle field operator $\psi(\vec{r})$ should be decomposed according to

$$
\psi(\vec{r})=\phi(\vec{r})+\sum_{j}\left(u_{j}(\vec{r}) \alpha_{j}+v_{j}^{*}(\vec{r}) \alpha_{j}^{\dagger}\right)
$$

where $\phi(\vec{r})=\langle\psi(\vec{r})\rangle$ is the order parameter, and the operators $\alpha_{j}^{\dagger}\left(\alpha_{j}\right)$ create (annihilate) the elementary excitations; the functions $u_{j}(\vec{r})$ and $v_{j}(\vec{r})$ are subject to the normalization condition

$$
\int \mathrm{d}^{3} r\left(\left|u_{j}(\vec{r})\right|^{2}-\left|v_{j}(\vec{r})\right|^{2}\right)=1 .
$$

The number of particles "out of the condensate" then is determined by [19]

$$
N_{\mathrm{ex}}=\int \mathrm{d}^{3} r\left(\psi^{\dagger}(\vec{r})-\phi^{*}(\vec{r})\right)(\psi(\vec{r})-\phi(\vec{r})),
$$

and, thus, is linked directly to the order parameter $\phi(\vec{r})$. When the interparticle interaction is characterized by a positive $s$-wave scattering length $a_{\mathrm{sc}}$ and the volume $V$ is sufficiently large, the order parameter tends to a constant function in the interior of $V$, thus resembling the constant density associated with the "periodic" single-particle ground state, except within distances of the order of the healing length

$$
\ell=\left(8 \pi a_{\mathrm{sc}} N / V\right)^{-1 / 2}
$$

from the boundaries, where $\phi(\vec{r})$ smoothly approaches zero [21]. Therefore, it is to be expected that the condensate fluctuations and the higher cumulants for an interacting gas in a box with Dirichlet boundary conditions and linear extension $L$ do approach the "periodic" result when $L \gg \ell$, or

$$
8 \pi N \frac{a_{\mathrm{sc}}}{L} \gg 1,
$$

so that the above findings for the ideal gas would have to be regarded as a pathology of the noninteracting system. However, at the moment this surmise should be taken as an educated guess only; in view of the principal importance of the condensate fluctuations-after all, these fluctuations determine the "line width of an atom laser" [22], or, more generally, the coherence properties of Bose-Einstein condensates at finite temperatures $[23,24]$ - an analytical proof is required. 
It should also be noted that it might be possible to violate the condition (23) experimentally, either by working with spin-polarized hydrogen that features an unusually small triplet scattering length [25], or by tuning the scattering length close to zero by means of a Feshbach resonance [26].
Such very weakly interacting Bose condensates, which fall into the regime between the ideal gas and the Bogoliubov gas, should exhibit particularly interesting statistical properties, and remain at least partly susceptible to boundary effects.
[1] F. London, Nature (London) 141, 643 (1938).

[2] F. London, Phys. Rev. 54, 947 (1938).

[3] M. Kac, Am. Math. Monthly 73, 1 (1966).

[4] M.O. Scully, Phys. Rev. Lett. 82, 3927 (1999).

[5] V.V. Kocharovsky, M.O. Scully, S.-Y. Zhu, and M.S. Zubairy, Phys. Rev. A 61, 023609 (2000).

[6] M. Holthaus, K.T. Kapale, V.V. Kocharovsky, and M.O. Scully, Physica A 300, 433 (2001).

[7] M. Fierz, Helv. Phys. Acta 29, 47 (1956).

[8] P. Navez, D. Bitouk, M. Gajda, Z. Idziaszek, and K. Rzążewski, Phys. Rev. Lett. 79, 1789 (1997).

[9] V.V. Kocharovsky, V1.V. Kocharovsky, and M.O. Scully, Phys. Rev. Lett. 84, 2306 (2000).

[10] V.V. Kocharovsky, Vl.V. Kocharovsky, and M.O. Scully, Phys. Rev. A 61, 053606 (2000).

[11] K. Kirsten and D.J. Toms, Phys. Rev. A 54, 4188 (1996).

[12] Handbook of Mathematical Functions, edited by M. Abramowitz and I. A. Stegun (Dover, New York, 1972).

[13] A. Voros, Commun. Math. Phys. 110, 439 (1987).

[14] K. Kirsten, J. Math. Phys. 32, 3008 (1991).
[15] K. Kirsten, J. Math. Phys. 35, 459 (1994).

[16] E. T. Whittaker and G. N. Watson, A Course of Modern Analysis (Cambridge University Press, Cambridge, 1969).

[17] R. K. Pathria, Statistical Mechanics (Butterworth-Heinemann, Oxford, 1996).

[18] C. Weiss and M. Wilkens, Opt. Express 1, 272 (1997).

[19] S. Giorgini, L.P. Pitaevskii, and S. Stringari, Phys. Rev. Lett. 80, 5040 (1998).

[20] F. Meier and W. Zwerger, Phys. Rev. A 60, 5133 (1999).

[21] V.L. Ginzburg and L.P. Pitaevskii, Zh. Eksp. Teor. Fiz. 34, 1240 (1958) [Sov. Phys. JETP 7, 858 (1958)].

[22] R. Graham, Phys. Rev. Lett. 81, 5262 (1998).

[23] S. Giorgini, Phys. Rev. A 61, 063615 (2000).

[24] R. Graham, Phys. Rev. A 62, 023609 (2000).

[25] M.J. Jamieson, A. Dalgarno, and M. Kimura, Phys. Rev. A 51, 2626 (1995).

[26] S. Inouye, M.R. Andrews, J. Stenger, H.-J. Miesner, D.M. Stamper-Kurn, and W. Ketterle, Nature (London) 392, 151 (1998). 